\title{
ON GENERALIZED RESOLVENTS
}

\author{
CONSTANTIN APOSTOL AND KEVIN CLANCEY
}

\begin{abstract}
Let $T$ be a bounded linear operator on a Hilbert space and $\rho_{I}(T)$ the Fredholm domain of $T$. It is shown that a generalized resolvent can be constructed for $T$ in $\rho_{F}(T)$ which verifies the resolvent identity except for an at most countable set of points which are close to the boundary of $\rho_{F}(T)$.
\end{abstract}

Let $T$ be a bounded linear operator on a Hilbert space $H$. In case the range of $T$ is a closed subspace of $H$, then an operator $F$ will be called a generalized inverse of $T$ when $F T$ is a projection onto the orthogonal complement of the kernel of $T$ and $T F$ is a projection onto the range of $T$. Unless $T$ is invertible, then a generalized inverse is not unique. Let $\mathcal{G}$ be a domain in the complex plane $\mathbf{C}$ such that for every $\lambda$ in $\mathcal{G}$, the operator $\lambda-T$ has closed range. An operator valued function $F$ defined on $\mathcal{G}$ is called a generalized inverse function for $T$ in $\mathcal{G}$ in case, for every $\lambda$ in $\mathcal{G}, F(\lambda)$ is a generalized inverse of $\lambda-T$. A generalized inverse function $F$ for $T$ on an open set $\mathcal{G}$ is said to verify the resolvent identity on $\mathcal{G}$, when for every pair $\lambda, \mu$ in a component of $\mathcal{Q}$

$$
F(\lambda)-F(\mu)=(\mu-\lambda) F(\lambda) F(\mu) .
$$

A continuous generalized inverse function, for an operator $T$ on an open set $\mathcal{G}$, which verifies the resolvent identity on $\mathcal{G}$ will be called a generalized resolvent on $\mathcal{G}$.

This note is concerned with the construction of generalized resolvents on open subsets of the Fredholm domain of a bounded operator $T$. Recall that an operator $T$ is called semi-Fredholm in case $T$ has closed range and the dimension of at least one of $\operatorname{ker}(T)$ or $\operatorname{ker}\left(T^{*}\right)$ is finite; here, $\operatorname{ker}$ denotes kernel and $T^{*}$ is the adjoint of $T$. If $T$ has closed range and both $\operatorname{ker}(T)$ and $\operatorname{ker}\left(T^{*}\right)$ are finite dimensional, then $T$ is called a Fredholm operator. The semi-Fredholm domain of $T$ is the set $\rho_{s-F}(T)=\{\lambda \in \mathbf{C}: \lambda-T$, is semi-Fredholm $\}$ and the Fredholm domain of $T$ is the set $\rho_{F}(T)=\{\lambda \in \mathbf{C}$ : $\lambda-T$ is Fredholm $\}$.

There is one obvious obstruction to constructing a generalized resolvent for $T$ on all of $\rho_{s-F}(T)$. In $\rho_{s-F}(T)$ there is an at most countable set where the function

$$
m(\lambda)=\text { minimum dimension }\left[\operatorname{ker}(\lambda-T), \operatorname{ker}(\lambda-T)^{*}\right]
$$

is discontinuous [3, Proposition 2.6], [5], [6]. This set will be denoted by $\rho_{s-F}^{s}(T)$ and is referred to as the set of singular points in the semi-Fredholm

Received by the editors December 13, 1974 and, in revised form, July 21, 1975. AMS (MOS) subject classifications (1970). Primary 47A10; Secondary 47A25. 
domain. The singular points in the Fredholm domain $\rho_{s-F}^{s}(T) \cap \rho_{F}(T)$ will be denoted by $\rho_{F}^{s}(T)$. The set $\rho_{s-F}^{s}(T)$ does not accumulate in $\rho_{s-F}(T)$ (see, e.g., [3], [5], [6]) and it is easy to show $\rho_{r}(T) \cap \rho_{s-F}^{s}(T)=\varnothing$. The complementary set $\rho_{s-F}^{r}(T)=\rho_{s-F}(T) \backslash \rho_{s-F}^{s}(T)\left(\rho_{F}^{r}(T)=\rho_{F}(T) \backslash \rho_{F}^{s}(T)\right)$ is called the set of regular points in the semi-Fredholm (Fredholm) domain. Obviously, there does not exist a continuous generalized inverse function in a neighborhood of a point $\lambda \in \rho_{s-F}^{s}(T)$.

The notation bdry $\mathcal{G}$ will be used for the boundary of a subset of $\mathbf{C}$ and $\operatorname{dist}\left(S, S^{\prime}\right)$ will denote the Hausdorff distance between two bounded sets $S$, $S^{\prime}$ in $\mathbf{C}$. In other words,

$$
\operatorname{dist}\left(S, S^{\prime}\right)=\max \left[\sup _{\lambda \in S^{\prime}}[\operatorname{distance}(\lambda, S)], \sup _{\lambda \in S}\left[\operatorname{distance}\left(\lambda, S^{\prime}\right)\right]\right] .
$$

The main result to be established here is

THEOREM 1. Let $T$ be a bounded operator on $H$ and let $\varepsilon>0$. There exists a generalized resolvent on $\rho_{F}^{r}(T)$ except for an at most countable set $S$ which does not accumulate in $\rho_{F}(T)$. Moreover, $\operatorname{dist}\left(S\right.$, bdry $\left.\rho_{F}(T)\right)<\varepsilon$.

There are several papers in the literature which contain results similar in spirit to the above theorem. In [8] P. Saphar obtains the above theorem (in the generality of operators on a Banach space) with the conclusion

$$
\text { “dist }\left(S, \text { bdry } \rho_{F}(T)\right)<\varepsilon "
$$

replaced by

$$
\text { “dist }\left(S, \text { bdry } \rho_{F}^{r}(T)\right)<\varepsilon " .
$$

Also Shapiro and Schechter [9] construct generalized resolvents on $\rho_{F}^{r}(T)$, minus a countable set $S$ for operators $T$ acting on a Banach space. These authors do not make any attempt to push the set $S$ out near the boundary of $\rho_{F}(T)$.

It is clear that a generalized resolvent for $T$ defined on an open set $\mathcal{G}$ is an analytic generalized inverse function for $T$ in $\mathcal{G}$. On the other hand, not every analytic generalized inverse function defined on an open set $\mathcal{G}$ verifies the resolvent identity on $\mathcal{G}$. Let $\rho_{r}(T)\left(\rho_{l}(T)\right)$ denote the set of complex $\lambda$, where $\lambda-T$ has a right (left) inverse. Allan [1], [2] has shown that there exists an analytic right (left) inverse function for $T$ in $\rho_{r}(T)\left(\rho_{l}(T)\right)$. This fact can be used to construct an analytic generalized inverse function for an operator $T$ in $\rho_{s-F}^{r}(T)$ [4].

Using the result in Theorem 1 it is possible to construct a "generalized spectral projection" associated with any finite subset $\sigma$ of $\rho_{F}^{s}(T)$. This leads to a decomposition of the operator $T$ as a direct sum $T_{1} \oplus T_{2}$ (not necessarily an orthogonal sum), where $T_{1}$ contains the singular points $\sigma$ as isolated eigenvalues in its spectrum and the operator $T_{2}$ satisfies $\rho_{F}^{r}\left(T_{2}\right)=\rho_{F}^{r}(T) \cup \sigma$. The reader is referred to [4] for further details.

1. Preliminaries. This section begins with a few basic lemmas:

Lemma 1. Let $T$ be an operator on $H$ and let $H_{1}, H_{2}$ be closed subspaces of H. Assume that relative to the orthogonal decomposition $H=H_{1} \oplus H_{2}$ the 
operator $T$ has the $2 \times 2$ matrix representation

$$
T=\left(\begin{array}{ll}
A & C \\
0 & B
\end{array}\right) .
$$

Let $\mathcal{G}$ be an open set on which both $A, B$ have right resolvents $R_{1}, R_{2}$ respectively. Then

$$
R=\left(\begin{array}{cc}
R_{1} & R_{1} C R_{2} \\
0 & R_{2}
\end{array}\right)
$$

defines a right resolvent of $T$ on $\mathcal{Q}$.

ProOF. Direct computation.

Lemma 2. Let $T$ be a bounded operator on $H$ and let $\dot{\mathcal{G}}_{1}$ be a connected open set in $\rho_{r}(T)$ such that dimension $(\operatorname{ker}(\lambda-T)) \equiv 1, \lambda \in \mathcal{G}_{1}$. For any $\varepsilon>0$ there is a right resolvent $R$ of $T$ on $\mathcal{G}_{1}$ except for an at most countable set $S$, which does not accumulate in $\mathcal{G}_{1}$, and satisfies $\operatorname{dist}\left(S\right.$, bdry $\left.\mathcal{G}_{1}\right)<\varepsilon$.

Proof. There is a vector $y \in H$ such that the orthogonal projection $P_{\text {ker }(\lambda-T)}$ onto the space $\operatorname{ker}(\lambda-T)$, satisfies $P_{\operatorname{ker}(\lambda-T)} y \neq 0$ for all $\lambda \in \mathcal{G}_{1}$ except for an at most countable set $S$ which does not accumulate in $\mathcal{G}_{1}$ and such that $\operatorname{dist}\left(S\right.$, bdry $\left.\mathcal{G}_{1}\right)<\varepsilon$ (for a proof see [3, Proposition 1.8]). Fix $\lambda_{0} \in \mathcal{G}_{1} \backslash S$ and let $\varphi$ be a nonzero vector in $\operatorname{ker}\left(\lambda_{0}-T\right)$. If $R_{0}$ is a fixed right inverse of $\lambda_{0}-T$, then any right inverse of $\lambda_{0}-T$ is of the form $R_{f}=R_{0}+\langle, f\rangle \varphi$, where $f \in H$. There is a choice of $f_{0} \in H$ such that $y \perp$ Range $R_{f_{0}}$.

In order to see this observe the orthogonal complement of the range $R_{f}$ is the null space of $R_{0}^{*}+\langle\cdot, \varphi\rangle f$. Now $R_{0}^{*} y+\langle y, \varphi\rangle f_{0}=0$, when $f_{0}=$ $-\langle y, \varphi\rangle^{-1} R_{0}^{*} y$. This last vector is well defined since $P_{\operatorname{ker}\left(\lambda_{0}-T\right)} y \neq 0$ and, therefore, $\langle y, \varphi\rangle \neq 0$.

For $\lambda \in \mathcal{G}_{1}$ the following identity holds:

$$
(\lambda-T) R_{f_{0}}=\left(\left(\lambda-\lambda_{0}\right) R_{f_{0}}+I\right) .
$$

This shows that $\lambda \rightarrow-\left(\lambda-\lambda_{0}\right)^{-1}$ is a mapping of $\mathcal{G}_{1}$ into the component of $\rho_{F}\left(R_{f_{0}}\right)$ which contains the point at infinity. Also, for $\lambda \in \mathcal{G}_{1}$, the Fredholm index of $\left(\lambda-\lambda_{0}\right) R_{f_{0}}+I$ is zero. Suppose, for some $\lambda_{1} \in \mathcal{G}_{1} \backslash S$, that $\operatorname{ker}\left[\left(\lambda_{1}-\lambda_{0}\right) R_{f_{0}}+I\right] \neq(0)$. Then from (2) it follows that, for some $x \neq 0$, $\left(\lambda_{1}-T\right) R_{f_{0}} x=0$. In this case, $R_{f_{0}} x \in \operatorname{ker}\left(\lambda_{1}-T\right)$ and since this last space is one dimensional, $\operatorname{ker}\left(\lambda_{1}-T\right) \subset$ Range $R_{f_{0}}$. This contradicts $y \perp$ Range $R_{f_{0}}$ and $P_{\operatorname{ker}\left(\lambda_{1}-T\right)} y \neq 0$. It is now clear that, for $\lambda$ in $\mathcal{G}_{1} \backslash S$, the operator $\left(\lambda-\lambda_{0}\right) R_{f_{0}}+I$ is invertible. The operator valued function $R(\lambda)=$ $R_{f_{0}}\left(\left(\lambda-\lambda_{0}\right) R_{f_{0}}+I\right)^{-1}$ is a right resolvent for $T$ in $\mathcal{G}_{1} \backslash S$. This completes the proof.

LEMMA 3. Let $T$ be a bounded operator on $H$ and let $\mathcal{G}_{n}$ be an open connected subset in $\rho_{r}(T)$. Assume that dimension $(\operatorname{ker}(\lambda-T))=n$, for $\lambda \in \mathcal{G}_{n}$. Then for any $\varepsilon>0$, there is a right resolvent for $T$ on $\mathcal{G}_{n}$ except for an at most countable set $S \subset \mathcal{G}_{n}$ which does not accumulate in $\mathcal{G}_{n}$, such that $\operatorname{dist}\left(S, \operatorname{bdry} \mathcal{G}_{n}\right)<\varepsilon$.

Proof. The proof proceeds by induction on $n$. The result is clear if $n=0$ 
for then $\mathcal{G}_{n}$ is a subset of the resolvent set of $T$. The case $n=1$ is contained in the preceding lemma. Suppose the result has been obtained in case $n=k-1$. Let $\mathcal{G}_{k}$ be a connected open subset of $\rho_{r}(T)$ such that dimension $\operatorname{ker}(\lambda-T)=k, \lambda \in \mathcal{G}_{k}$. For any $\varepsilon>0$ there is a vector $y \in H$ for which $P_{\operatorname{ker}(\lambda-T)} y \neq 0$, for all $\lambda \in \mathcal{G}_{k} \backslash S^{\prime}$; where $S^{\prime}$ is an at most countable set which does not accumulate in $\mathcal{G}_{k}$ and satisfies $\operatorname{dist}\left(S^{\prime}\right.$, bdry $\left.\mathcal{G}_{k}\right)<\varepsilon$ (see [3, Proposition 1.8]). Let $Y_{\lambda}=\operatorname{ker}(\lambda-T) \cap\{y\}^{\perp}$, for $\lambda \in \mathcal{G}_{k}$, and let $Y=$ c.l.m. ${ }_{\lambda \in \mathcal{S}_{k}}\left\{Y_{\lambda}\right\}$; here, c.l.m. is an abbreviation for closed linear manifold. Obviously, $T Y \subset Y$ and relative to the decomposition $H=Y \oplus Y^{\perp}$,

$$
T=\left(\begin{array}{ll}
T_{Y} & * \\
0 & T_{Y^{\perp}}
\end{array}\right)
$$

here, $T_{Y}$ is the restriction of $T$ to $Y$ and $T_{Y^{\perp}}$ is the compression of $T$ to $Y^{\perp}$. It is easy to establish that $\lambda-T_{Y}$ is onto for $\lambda \in \mathcal{G}_{k}$ and clearly $\left(\lambda-T_{Y^{\perp}}\right)$ is onto for $\lambda \in \mathcal{G}_{k}$. It follows that for $\lambda \in \mathcal{G}_{k} \backslash S^{\prime}$, dimension $\operatorname{ker}\left(\lambda-T_{Y}\right)$ $=k-1$ and dimension $\operatorname{ker}\left(\lambda-T_{Y^{\perp}}\right)=1$. The induction hypothesis can be combined with Lemma 1 and Lemma 2 to complete the proof.

Following [3] we introduce the notations

$$
\begin{aligned}
& H_{r}(T)=\text { c.l.m. }{ }_{\cdot \lambda \in \rho_{s-F}^{r}(T)} \operatorname{ker}(\lambda-T), \\
& H_{l}(T)=\text { c.l.m. }{ }_{\cdot \lambda \in \rho_{s}^{r}(T)} \operatorname{ker}(\lambda-T)^{*}, \\
& H_{0}(T)=H \ominus\left(H_{r}(T) \oplus H_{l}(T)\right) .
\end{aligned}
$$

The proof that $H_{r}(T)$ and $H_{l}(T)$ are orthogonal subspaces appears in [3].

Relative to the decomposition $H=H_{r}(T) \oplus H_{0}(T) \oplus H_{l}(T)$ the operator $T$ has the $3 \times 3$ matrix form

$$
T=\left(\begin{array}{lll}
T_{r} & A & B \\
0 & T_{0} & C \\
0 & 0 & T_{l}
\end{array}\right)
$$

The relevant spectral properties of $T_{r}, T_{0}, T_{l}$ are:

(i) $\rho_{s-F}(T) \subset \rho_{r}\left(T_{r}\right) \cap \rho_{l}\left(T_{l}\right)$,

(ii) $\rho_{s-F}^{r}(T) \subset \rho\left(T_{0}\right)$ (the resolvent set of $T_{0}$ ),

(iii) $\rho_{s-F}^{s}(T)$ is a subset of the isolated eigenvalues of $T_{0}$ which have finite algebraic multiplicity.

The proofs of the inclusions (i)-(iii) appear in [3].

Next let $\mathcal{G}$ be an open set and $T$ an operator having the $3 \times 3$ matrix form (3) relative to the decomposition $H=H_{r}(T) \oplus H_{0}(T) \oplus H_{l}(T)$. Assume further that $T_{r}$ has a right inverse function $R$ in $\mathcal{G}, T_{l}$ has a left inverse function $L$ in $\mathcal{G}$ and that $\mathcal{G} \subset \rho\left(T_{0}\right)$.

For $\lambda$ in $\mathcal{G}$, set

(4) $F(\lambda)=\left(\begin{array}{ccc}R(\lambda) & R(\lambda) A R\left(\lambda: T_{0}\right) & R(\lambda)\left[A R\left(\lambda: T_{0}\right) C+B\right] L(\lambda) \\ 0 & R\left(\lambda: T_{0}\right) & R\left(\lambda: T_{0}\right) C L(\lambda) \\ 0 & 0 & L(\lambda)\end{array}\right)$;

here, $R\left(\lambda: T_{0}\right)=\left(\lambda-T_{0}\right)^{-1}$.

The operator valued function has the following properties [4]:

(a) $F$ is a generalized inverse function for $F$ in $\mathcal{G}$. 
(b) If $R, L$ are analytic in $\mathcal{G}$, then $F$ is an analytic generalized inverse function for $T$ in $\mathcal{G}$.

(c) If $R, L$ are right and left resolvents of $T_{r}, T_{l}$, respectively, then $F$ is a generalized resolvent of $T$ in $\mathcal{G}$.

2. Proof of Theorem 1. Let $\varepsilon>0$. It suffices to construct a generalized resolvent for $T$ on each component $\mathcal{G}$ of $\rho_{F}^{r}(T)$ except for an at most countable set $S$ which does not accumulate in $\mathcal{G}$ and satisfies $\operatorname{dist}(S, \operatorname{bdry} \mathcal{G})$ $<\varepsilon$. For such a $\mathcal{G}$ we have $\mathcal{G} \subset \rho_{r}\left(T_{r}\right) \cap \rho_{l}\left(T_{l}\right)$. Moreover,

$$
\begin{aligned}
\text { dimension } \operatorname{ker}\left(\lambda-T_{r}\right) & \equiv n<\infty, \\
\text { dimension } \operatorname{ker}\left(\lambda-T_{l}\right)^{*} & \equiv m<\infty \quad \text { on } \mathcal{G} .
\end{aligned}
$$

It follows from Lemma 3 that we can construct a right resolvent $R$ for $T_{r}$ in all of $\mathcal{G}$ except for an at most countable set $S^{\prime}$ which does not accumulate in $\mathcal{G}$ and satisfies $\operatorname{dist}\left(S^{\prime}\right.$, bdry $\left.\mathcal{G}\right)<\varepsilon$. The same argument applied to $T_{l}^{*}$ in $\mathcal{G}^{*}$ implies the existence of a left resolvent $L$ for $T_{l}$ in all of $\mathcal{G}$ except for an at most countable set $S^{\prime \prime}$ which does not accumulate in $\mathcal{G}$ and satisfies $\operatorname{dist}\left(S^{\prime \prime}\right.$, bdry $\left.\mathcal{G}\right)<\varepsilon$.

From the inclusion $\mathcal{G} \subset \rho\left(T_{0}\right)$ it follows that the function $F$ defined by (4) is a generalized resolvent for $T$ in $\mathcal{G}$ except for the at most countable set $S=S^{\prime} \cup S^{\prime \prime}$, where $S$ does not accumulate in $\mathcal{G}$ and satisfies $\operatorname{dist}(S$, bdry $\mathcal{G})$ $<\varepsilon$. This completes the proof.

REMARK. It would be possible to extend the above argument to construct a generalized resolvent in all of $\rho_{r}(T)$ if the following question has an affirmative answer.

Question. Let $\mathcal{G}$ be an open connected subset in $\rho_{r}(T)$. Assume

$$
\text { dimension } \operatorname{ker}(\lambda-T)=1 \text {, for all } \lambda \in \mathcal{G} \text {. }
$$

Does there exist a $y$ in $H$ such that $P_{\operatorname{ker}(\lambda-T)} y \neq 0$, for all $\lambda \in \mathcal{G}$ ?

In connection with the above question we refer the reader to [8] and the example of A. Douady. In [8, p. 244] an example is given of a holomorphic Hilbert space valued function on the unit disc $D$ with the following properties: (i) For each compact subset $K \subset D$, there is an $x \in H$ such that $(h(z)$, $x$ ) has no zeroes on $K$. (ii) For all $y \in H$, the function $(h(z), y)$ has a zero in $D$.

ACKNOWLedgement. The authors would like to thank the referee for the references to the work of P. Saphar.

\section{REFERENCES}

1. G. R. Allan, On one-sided inverses in Banach algebras of holomorphic vector-valued functions, J. London Math. Soc. 42 (1967), 463-470. MR 35 \# 5939.

2. _ Holomorphic vector-valued functions on a domain of holomorphy, J. London Math. Soc. 42 (1967), 509-513. MR 35 \#5940.

3. C. Apostol, The correction by compact perturbation of the singular behavior of operators, Rev. Roumaine Math. Pures Appl. (to appear).

4. C. Apostol and K. Clancey, Generalized inverses and spectral theory, Trans. Amer. Math. Soc. 215 (1976), 293-300.

5. I. C. Gohberg and M. G. Kreìn, The basic propositions on defect numbers, root numbers and indices of linear operators, Uspehi Mat. Nauk 12 (1957), no. 2 (74), 43-118; English transl., Amer. Math. Soc. Transl. (2) 13 (1960), 185-264. MR 20 \#3459; 22 \#3984. 
6. T. Kato, Perturbation theory for linear operators, Die Grundlehren der math. Wissenschaften, Band 132, Springer-Verlag, New York, 1966. MR 34 \#324.

7. P. Saphar, Contributions à l'étude des applications linéaires dans un espace de Banach, Bull. Soc. Math. France 92 (1964), 363-384. MR 32 \#4549.

8. - Sur les applications linéaires dans un espace de Banach. II, Ann. Sci. Ecole Norm. Sup. (3) 82 (1965), 205-240. MR 35 \#2164.

9. J. Shapiro and M. Schechter, A generalized operational calculus developed from Fredholm operator theory, Trans. Amer. Math. Soc. 175 (1973), 439-467. MR 47 \#2406.

INSTITUTE OF MATHEMATICS, BUCHAREST, ROMANIA

Department of Mathematics, University of Georgia, Athens, Georgia 30602 